\title{
ON THE CONVERGENCE OF SOME ITERATION PROCESSES IN UNIFORMLY CONVEX BANACH SPACES
}

\section{J. GWINNER}

\begin{abstract}
For the approximation of fixed points of a nonexpansive operator $T$ in a uniformly convex Banach space $E$ the convergence of the Mann-Toeplitz iteration $x_{n+1}=\alpha_{n} T\left(x_{n}\right)+\left(1-\alpha_{n}\right) x_{n}$ is studied. Strong convergence is established for a special class of operators $T$. Via regularization this result can be used for general nonexpansive operators, if $E$ possesses a weakly sequentially continuous duality mapping. Furthermore strongly convergent combined regularization-iteration methods are presented.
\end{abstract}

Throughout this note, let $(E,|\cdot|)$ be a uniformly convex Banach space, let $C$ be a nonempty closed convex subset of $E$. Let $T: C \rightarrow C$ denote a nonexpansive operator, i.e. $|T(x)-T(y)| \leqslant|x-y|$ holds for all $x, y \in C$. To approximate a fixed point of $T$ we define the following iterative method (Mann-Toeplitz process) by

$$
x_{1} \in C, \quad x_{n+1}=\alpha_{n} T\left(x_{n}\right)+\left(1-\alpha_{n}\right) x_{n}, \quad \alpha_{n} \in[0,1](n \geqslant 1) .
$$

We make the assumptions that $\sum_{n=1}^{\infty} \alpha_{n}=\infty$ and $\alpha_{n} \in[0, b]$ with $b \in(0,1)$ for almost all positive integers $n$.

Let us recall that any mapping $J: E \rightarrow E^{*}$ which fulfills

$$
(J(u), u)=|J(u)| \cdot|u|, \quad|J(u)|=|u|
$$

for all $u \in E$ is termed a duality mapping. We verify easily (see also [3, Theorem 8.9]) that the nonexpansive operator $T$ satisfies

$$
(x-y-T(x)+T(y), J(x-y)) \geqslant 0
$$

for all $x, y \in C$. This means that the operator $S:=I-T$ is accretive. Now we call an operator $S: C \rightarrow E \phi$-accretive if there exists a function $\varphi$ : $[0, \infty) \rightarrow[0, \infty)$ strictly increasing with $\varphi(0)=0$, and $\lim _{t \rightarrow \infty} \varphi(t)=\infty$ such that (cf. [1] for monotone operators)

$$
\begin{aligned}
(S(x)-S(y), J(x-y)) \geqslant[\varphi(|x|)-\varphi(|y|)] \cdot[|x|-|y|] & \\
& \forall x, y \in C .
\end{aligned}
$$

If $S$ satisfies the stronger condition

Received by the editors November 17, 1976 and, in revised form, August 22, 1977.

AMS (MOS) subject classifications (1970). Primary 47H15, $65 \mathrm{~J} 05$.

Key words and phrases. Nonexpansive, $\varphi$-accretive, duality mapping, fixed point, iteration, regularization. 


$$
(S(x)-S(y), J(x-y)) \geqslant \varphi(|x-y|) \cdot|x-y| \quad \forall x, y \in C,
$$

then $S$ is called uniformly $\varphi$-accretive.

THEOREM 1. Let the fixed point set $F$ of the operator $T$ be nonempty. Suppose the operator $S=I-T$ is $\varphi$-accretive. Then the Mann-Toeplitz sequence $\left\{x_{n}\right\}$ converges strongly to the unique fixed point $p \in F$.

Proof. Since for any fixed $p \in F$

$$
\left|x_{n+1}-p\right| \leqslant\left|x_{n}-p\right| \text {, }
$$

the sequence $\left\{x_{n}\right\}$ is bounded. Now assume $p_{1}, p_{2}$ belong to $F$. By (2) it follows that

$$
\left[\varphi\left(\left|p_{1}\right|\right)-\varphi\left(\left|p_{2}\right|\right)\right] \cdot\left[\left|p_{1}\right|-\left|p_{2}\right|\right]=0,
$$

therefore $\left|p_{1}\right|=\left|p_{2}\right|$. On the other hand $F$ is convex, and is contained in the uniformly convex space $E$. So $F$ reduces to a single point $p$.

According to a result of Ishikawa [7, Lemma 2] $S\left(x_{n}\right)$ converges strongly to zero, and by a theorem due to Browder [3, Theorem 8.4, p. 103] the sequence $\left\{x_{n}\right\}$ converges weakly to the unique fixed point $p$. Since $J$ is a bounded operator, the sequence $\left\{J\left(x_{n}-p\right)\right\}$ remains bounded. Hence (2) implies that

$$
\left[\varphi\left(\left|x_{n}\right|\right)-\varphi(|p|)\right] \cdot\left[\left|x_{n}\right|-|p|\right] \rightarrow 0 \quad(n \rightarrow \infty) .
$$

It follows easily (cf. [1, p. 61]) that $\left|x_{n}\right| \rightarrow|p|$. This yields the claimed norm convergence of the sequence $\left\{x_{n}\right\}$ in the uniformly convex space $E$.

Since strictly contractive operators are uniformly $\varphi$-accretive, Theorem 3 contains a result in [5, Theorem 1]. Let us note that in a Hilbert space $E$ the gradient $f^{\prime}$ of a Gateaux differentiable, convex functional $f$ is $\varphi$-accretive, if

$$
f((x+y) / 2) \leqslant \frac{1}{2} f(x)+\frac{1}{2} f(y)-[\varphi(|x|)-\varphi(|y|)] \cdot[|x|-|y|]
$$

is valid for all $x, y \in C$. This fact follows from the estimate

$$
\left(f^{\prime}(x)-f^{\prime}(y), x-y\right) \geqslant 2[f(x)+f(y)-2 f((x+y) / 2)] .
$$

Furthermore Theorem 1 remains true, if the inequality (2) is only assumed to hold for all $x \in C$ and all $p \in F$, i.e. if

$$
(x-T(x), J(x-p)) \geqslant[\varphi(|x|)-\varphi(|p|)] \cdot[|x|-|p|] \quad \forall x \in C, \forall p \in F
$$

is assumed. Operators $T: C \rightarrow C$ that satisfy for any $x, y \in C$

$$
\begin{aligned}
|T(x)-T(y)| \leqslant & a_{1}|x-T(x)|+a_{2}|y-T(y)| \\
& +a_{3}|x-y|+a_{4}|x-T(y)|+a_{5}|y-T(x)|
\end{aligned}
$$

with $a_{i} \geqslant 0(i=1, \ldots, 5)$ and $\sum_{i=1}^{5} a_{i} \leqslant 1$ belong to this class, provided

$$
2 a_{1}+a_{3}+a_{4}+a_{5}<1
$$

holds.

Even Theorem 1 can be applied to general nonexpansive operators $T$, for then the operators $S_{\varepsilon}=(1-\varepsilon)(I-T)+\varepsilon R(\varepsilon>0)$ inherit the (uniform) $\varphi$-accretiveness from the "regularization operator" $R$. This observation motivates the following study of the regularization method involved. 
Theorem 2. Let the fixed point set $F$ of $T$ in $C$ be nonempty. Let $R: C \rightarrow E$ be a continuous, bounded operator. Suppose $R$ is uniformly $\varphi$-accretive with respect to an odd, weakly sequentially continuous duality mapping $J: E \rightarrow E^{*}$. Choose positive reals $\delta_{k}$, and $\varepsilon_{k} \in(0,1)$ with $\lim _{k \rightarrow \infty} \varepsilon_{k}=0$, and $\lim _{k \rightarrow \infty} \delta_{k} \varepsilon_{k}^{-1}$ $=0$. If the approximate solutions $\tilde{y}_{k} \in C$ satisfy

$$
\left|\left(1-\varepsilon_{k}\right)(I-T)\left(\tilde{y}_{k}\right)+\varepsilon_{k} R\left(\tilde{y}_{k}\right)\right| \leqslant \delta_{k},
$$

then the sequence $\left\{\tilde{y}_{k}\right\}$ converges strongly to a fixed point $\hat{p}$, which is uniquely determined by the variational inequality

$$
(R(\hat{p}), J(\hat{p}-p)) \leqslant 0 \quad \forall p \in F .
$$

Proof. Let $p \in F$, and set

$$
\beta_{k}=\left(\left(1-\varepsilon_{k}\right)(I-T)\left(\tilde{y}_{k}\right)+\varepsilon_{k} R\left(\tilde{y}_{k}\right), J\left(\tilde{y}_{k}-p\right)\right) .
$$

We notice that $\left|\beta_{k}\right| \leqslant\left|\tilde{y}_{k}-p\right| \delta_{k}$. Since $I-T$ is accretive, it follows

$$
\left(R\left(\tilde{y}_{k}\right), J\left(\tilde{y}_{k}-p\right)\right) \leqslant \beta_{k} \varepsilon_{k}^{-1} .
$$

Let us prove the boundedness of the sequence $\left\{y_{k}\right\}$. On account of (5), (3) we conclude

$$
\begin{aligned}
\beta_{k} \varepsilon_{k}^{-1}+|R(p)| \cdot\left|\tilde{y}_{k}-p\right| & \geqslant \beta_{k} \varepsilon_{k}^{-1}-\left(R(p), J\left(\tilde{y}_{k}-p\right)\right) \\
& \geqslant\left(R\left(\tilde{y}_{k}\right)-R(p), J\left(\tilde{y}_{k}-p\right)\right) \\
& \geqslant \varphi\left(\left|\tilde{y}_{k}-p\right|\right) \cdot\left|\tilde{y}_{k}-p\right| .
\end{aligned}
$$

We may assume without loss of generality that $\left|\tilde{y}_{k}-p\right|$ is positive, and hence we obtain

$$
\delta_{k} \varepsilon_{k}^{-1}+|R(p)| \geqslant \varphi\left(\left|\tilde{y}_{k}-p\right|\right) .
$$

The boundedness of $\left\{\tilde{y}_{k}\right\}$ is immediate, and with a constant $c_{p}$, dependent only on $p \in F,(5)$ reads

$$
\left(R\left(\tilde{y}_{k}\right), J\left(\tilde{y}_{k}-p\right)\right) \leqslant c_{p} \delta_{k} \varepsilon_{k}^{-1} .
$$

As $R$ is a bounded operator, $\varepsilon_{k} R\left(\tilde{y}_{k}\right)$ converges to zero. Since the nonexpansive operator $T$ is also bounded, and $\delta_{k} \rightarrow 0$, we conclude that $(I-T)\left(\tilde{y}_{k}\right)$ converges strongly to zero. By a theorem due to Browder [3, Theorem 8.4] all weak limit points of $\left\{\tilde{y}_{k}\right\}$, which exist by the boundedness of $\left\{\tilde{y}_{k}\right\}$, belong to $F$. Let $\tilde{y}=\mathrm{w}-\lim _{i \rightarrow \infty} \tilde{y}_{k_{i}}$; then (3) and (6) imply that

$$
c_{\tilde{y}} \delta_{k_{i}} \varepsilon_{k_{i}}^{-1}-\left(R(\tilde{y}), J\left(\tilde{y}_{k_{i}}-\tilde{y}\right)\right) \geqslant \varphi\left(\left|\tilde{y}_{k_{i}}-\tilde{y}\right|\right) \cdot\left|\tilde{y}_{k_{i}}-\tilde{y}\right| .
$$

Since $J$ is weakly sequentially continuous, we see at once that $\tilde{y}=\lim _{i \rightarrow \infty} y_{k_{i}}$, and (6) results in the claimed inequality (4). Finally we have to show that this inequality uniquely determines $\hat{p} \in F$, thus proving the convergence of the entire sequence $\left\{\tilde{y}_{k}\right\}$. Fix some $p_{1}, p_{2} \in F$ that satisfy (4), then

$$
\left(R\left(p_{1}\right), J\left(p_{1}-p_{2}\right)\right) \leqslant 0, \quad-\left(R\left(p_{2}\right), J\left(p_{1}-p_{2}\right)\right) \leqslant 0 .
$$

The summation of both these inequalities yields $p_{1}=p_{2}$, since $R$ is uniformly $\varphi$-accretive. 
If the regularization operator $R$ is only $\varphi$-accretive, then similar but more involved arguments show that the sequence $\left\{\tilde{y}_{k}\right\}$ is bounded, every weak limit point of $\left\{\tilde{y}_{k}\right\}$ is also a strong limit point, and every limit point belongs to the fixed point set and satisfies (4). But as $J$ is not linear, unless $E$ is a Hilbert space, the set of points which fulfill (4) is generally not convex; therefore uniqueness cannot be obtained as in the proof of Theorem 1.

The approximate solutions $\tilde{y}_{k}$ can be constructed by finitely many MannToeplitz iterations for the operator $T_{k}=\left(1-\varepsilon_{k}\right) T+\varepsilon_{k}(I-R)$ by Theorem 1 , provided $I-R: C \rightarrow C$ is nonexpansive. If furthermore $C$ is bounded, fixed points of $T$ and of each $T_{k}$ exist.

The simplest regularization method is given by $R(x)=x-x^{0}, x^{0}$ fixed in $C$. In this case Reich [10, Corollary] has already established the strong convergence of the exact solutions $y_{k}=\left(1-\varepsilon_{k}\right) T\left(y_{k}\right)+\varepsilon_{k} x^{0}\left(\delta_{k}=0\right)$ to a fixed point of $T$ under similar conditions. In view of the inequality (4) which is achieved by regularization, other choices of $R$ should be taken into consideration.

Inspired by the work of Bruck [4], and Halpern [6] we combine in conclusion Mann-Toeplitz iteration and regularization to the following iteration process

$$
z_{1} \in C, z_{m+1}=\alpha_{m}\left(1-\varepsilon_{m}\right) T\left(z_{m}\right)+\alpha_{m} \varepsilon_{m} U\left(z_{m}\right)+\left(1-\alpha_{m}\right) z_{m} .
$$

Here we require that $U: C \rightarrow C$ is a strict contraction with contraction constant $q \in[0,1)$. Clearly $R=I-U$ is then uniformly $\varphi$-accretive with $\varphi(t)=(1-q) t$. Let us note that the choice $U(z)=z$, $z$ fixed in $C$, reduces (7) with $\varepsilon_{m}=\Theta_{m}\left(1+\Theta_{m}\right)^{-1}, \alpha_{m}=\lambda_{m}\left(1+\Theta_{m}\right)$ to the iteration method which is considered in [4, p. 123], and is also contained in the projection-iteration method of Bakusinskii and Poljak [2, Theorem 3D] for the solution of variational inequalities in Hilbert spaces.

The subsequent results hold in arbitrary Banach spaces $E$.

THEOREM 3. Let $C$ be a bounded closed convex subset of $E$. Suppose the sequence $\left\{y_{i}\right\}$ converges to a fixed point $p$ of $T$, where $y_{i}$ is given by

$$
y_{i}=\left(1-\varepsilon_{i}\right) T\left(y_{i}\right)+\varepsilon_{i} U\left(y_{i}\right)
$$

with $\varepsilon_{i} \in(0,1],\left\{\varepsilon_{i}\right\}$ monotonically decreasing to zero. If the two sequences $\left\{\varepsilon_{m}\right\}$ and $\left\{\alpha_{m}\right\}$, contained in $(0,1]$, satisfy with some strictly increasing sequence $\{m(k)\}$ of positive integers

$$
\begin{array}{r}
\liminf _{k \rightarrow \infty} \varepsilon_{m(k)} \sum_{j=m(k)}^{m(k+1)} \alpha_{j}>0, \\
\lim _{k \rightarrow \infty}\left[\varepsilon_{m(k)}-\varepsilon_{m(k+1)}\right] \cdot \sum_{j=m(k)}^{m(k+1)} \alpha_{j}=0,
\end{array}
$$

then the sequence $\left\{z_{m}\right\}$ generated by (7) converges to $p$.

Proof. We follow the pattern of proof in [4, pp. 117-119], but we dispense with inner product structure. 
Banach's fixed point theorem guarantees existence and uniqueness of each $y_{i}$. We calculate for $m>i \geqslant 1$

$$
\begin{aligned}
\left|z_{m}-y_{i}\right|= & \mid \alpha_{m-1}\left(1-\varepsilon_{m-1}\right) T\left(z_{m-1}\right)+\alpha_{m-1} \varepsilon_{m-1} U\left(z_{m-1}\right) \\
\leqslant & \left(1-\alpha_{m-1}\right)\left|z_{m-1}-y_{i}\right| \\
& +\alpha_{m-1} \mid\left(1-\varepsilon_{m-1}\right) T\left(z_{m-1}\right)+\varepsilon_{m-1} U\left(z_{m-1}\right) \\
& \quad-\left(1-\varepsilon_{i}\right) T\left(y_{i}\right)-\varepsilon_{i} U\left(y_{i}\right) \mid \\
\leqslant & {\left[1-\alpha_{m-1}+\alpha_{m-1}\left(1-\varepsilon_{i}\right)+\alpha_{m-1} \varepsilon_{i} q\right] \cdot\left|z_{m-1}-y_{i}\right| } \\
& +\alpha_{m-1}\left(\varepsilon_{i}-\varepsilon_{m-1}\right) \cdot\left|T\left(z_{m-1}\right)-U\left(z_{m-1}\right)\right| .
\end{aligned}
$$

Hence

$$
\left|z_{m}-y_{i}\right| \leqslant\left[1-\alpha_{m-1} \varepsilon_{i}(1-q)\right] \cdot\left|z_{m-1}-y_{i}\right|+\alpha_{m-1}\left(\varepsilon_{i}-\varepsilon_{m-1}\right) c,
$$

with some constant $c$, because $T$ and $U$ are self-mappings of the bounded set $C$. Since the exp function is convex and therefore $\exp (t)-1 \geqslant t$ holds, it follows that

$$
\left|z_{m}-y_{i}\right| \leqslant \exp \left[-\alpha_{m-1} \varepsilon_{i}(1-q)\right] \cdot\left|z_{m-1}-y_{i}\right|+c \alpha_{m-1}\left(\varepsilon_{i}-\varepsilon_{m-1}\right) .
$$

By induction we conclude

$$
\left|z_{m}-y_{i}\right| \leqslant \exp \left[-\varepsilon_{i}(1-q) \sum_{j=i}^{m-1} \alpha_{j}\right] \cdot\left|z_{i}-y_{i}\right|+c \sum_{j=i}^{m-1} \alpha_{j}\left(\varepsilon_{i}-\varepsilon_{j}\right) .
$$

On account of $\varepsilon_{i}-\varepsilon_{j} \leqslant \varepsilon_{i}-\varepsilon_{m}$ for $j \leqslant m$ we weaken this estimate to

$$
\left|z_{m}-y_{i}\right| \leqslant \exp \left[-(1-q) \varepsilon_{i} \sum_{j=i}^{m-1} \alpha_{j}\right] \cdot\left|z_{i}-y_{i}\right|+c\left(\varepsilon_{i}-\varepsilon_{m}\right) \sum_{j=i}^{m} \alpha_{j} .
$$

Starting with this inequality, which corresponds to inequality (12) in [4], one can easily adapt the arguments in [4, pp. 118-119] to conclude the proof. The details are omitted.

Examples of sequences $\left\{\alpha_{n}\right\}$ and $\left\{\varepsilon_{n}\right\}$ that satisfy both the conditions (9) and (10) are given by $\alpha_{n}=1 / n$, and $\varepsilon_{n}=1 / \log \log n$ for $n>2$, or by $\alpha_{n}=n^{-p}$ and $\varepsilon_{n}=n^{-q}$ for $n>2$, provided $0<p<1$ and $0<q<1-p$ holds (see Bruck [4, p. 125]). Also one can choose $\alpha_{n}=\lambda \in(0,1]$ fixed, and $\varepsilon_{n(k)}=\varepsilon_{n(k)+1}=\cdots=\varepsilon_{n(k+1)-1}=k^{1-p}$, where $n(k) \sim k^{p}$ and $p>1$. The resulting iteration process is then related to [6, Theorem 4]. Furthermore the choice $\alpha_{n}=\lambda, \varepsilon_{n}=n^{-p}\left(1+n^{-p}\right)^{-1}, p \in(0,1)$ with $n(k) \sim k^{r}, r=(1-$ $p)^{-1}$ leads to the example D of Theorem 3D with $P_{K}=I$ in [2].

Simpler sufficiency criteria for strong convergence are provided by

TheOREM 4. Let $C$ be a bounded closed convex subset of E. Suppose, the sequence $\left\{y_{i}\right\}$, given by (8), converges to a fixed point $p$ of $T$, where $\varepsilon_{i} \in(0,1]$ and $\lim _{i \rightarrow \infty} \varepsilon_{i}=0$. If the two sequences $\left\{\varepsilon_{i}\right\}$ and $\left\{\alpha_{i}\right\}$, contained in $(0,1]$, satisfy

$$
\sum_{i} \alpha_{i} \varepsilon_{i}=+\infty
$$




$$
\left|\varepsilon_{i-1}-\varepsilon_{i}\right| \cdot \varepsilon_{i}^{-1}=o\left(\alpha_{i} \varepsilon_{i}\right),
$$

then the sequence $\left\{z_{m}\right\}$ generated by (7) converges to $p$.

Proof. We simplify (11) to

$$
\delta_{m+1}:=\left|z_{m+1}-y_{m}\right| \leqslant\left[1-\alpha_{m} \varepsilon_{m}(1-q)\right]\left|z_{m}-y_{m}\right| \text {. }
$$

On the other hand we have

$$
\begin{aligned}
\left|y_{i}-y_{i-1}\right| \leqslant & \left(1-\varepsilon_{i}\right)\left|y_{i}-y_{i-1}\right|+\left|\varepsilon_{i-1}-\varepsilon_{i}\right| \cdot\left|T\left(y_{i-1}\right)\right|+\varepsilon_{i} q\left|y_{i}-y_{i-1}\right| \\
& +\left|\varepsilon_{i-1}-\varepsilon_{i}\right| \cdot\left|U\left(y_{i-1}\right)\right|
\end{aligned}
$$

hence with some constant $d$

$$
\left|y_{i}-y_{i-1}\right| \leqslant d \cdot\left|\varepsilon_{i-1}-\varepsilon_{i}\right| \varepsilon_{i}^{-1} .
$$

Thus we obtain with $\chi_{m}=(1-q) \alpha_{m} \varepsilon_{m}, \gamma_{m}=d \cdot\left|\varepsilon_{m-1}-\varepsilon_{m}\right| \varepsilon_{m}^{-1} \cdot \chi_{m}^{-1}$

$$
\delta_{m+1} \leqslant\left(1-\chi_{m}\right) \delta_{m}+\chi_{m} \gamma_{m} \text {, }
$$

and consequently for arbitrary $j \geqslant 0$

$$
\delta_{m+j+1} \leqslant\left(\prod_{i=m}^{m+j}\left(1-\chi_{i}\right)\right) \delta_{m}+\sum_{i=m}^{m+j}\left(\prod_{k=i+1}^{m+j}\left(1-\chi_{k}\right)\right) \chi_{i} \gamma_{i}
$$

By (12), $\Pi\left(1-\chi_{i}\right)$ diverges to zero. Since

$$
\sum_{i=m}^{m+j}\left(\prod_{k=i+1}^{m+j}\left(1-\chi_{k}\right)\right) \chi_{i} \leqslant 1
$$

for any $j$ and $\lim _{i \rightarrow \infty} \gamma_{i}=0$ by (13), a well-known theorem of Toeplitz (cf. [8, p. 75]) implies that the second term in (14) converges to zero $(j \rightarrow \infty)$, too. Thus we arrive at $\lim _{i \rightarrow \infty} \delta_{i}=0$.

This result is closely related to Theorem 3D in [2] and contains (choose $U(z)=y$ fixed, $\alpha_{i}=1$ fixed) a recent result of Lions [9, Theorem 1].

The author wishes to thank the referee for pointing out the references [2], [7].

\section{REFERENCES}

1. H. Brézis and M. Sibony, Méthodes d'approximation et d'itération pour les opérateurs monotones, Arch. Rational Mech. Anal. 28 (1967/68), 59-82. MR 36 \# 3177.

2. A. B. Bakusinskii and B. T. Poljak, On the solution of variational inequalities, Dokl. Akad. Nauk SSSR 219 (1974), 1038-1041 = Soviet Math. Dokl. 15 (1974), 1705-1710. MR 51 \# 13790.

3. F. E. Browder, Nonlinear operators and nonlinear equations of evolution in Banach spaces, Proc. Sympos. Pure Math., vol. 18, part II, Amer. Math. Soc., Providence, R.I., 1976. MR 53 \#8982.

4. R. E. Bruck, Jr., A strongly convergent iterative solution of $0 \in U(x)$ for a maximal monotone operator $U$ in Hilbert space, J. Math. Anal. Appl. 48 (1974), 114-126. MR 50 \# 14383.

5. J. C. Dunn, Convexity, monotonicity, and gradient processes in Hilbert space, J. Math. Anal. Appl. 53 (1976), 145-158.

6. B. Halpern, Fixed points of nonexpanding maps, Bull. Amer. Math. Soc. 73 (1967), 957-961. MR 36 \#2022.

7. S. Ishikawa, Fixed points and iteration of a nonexpansive mapping in a Banach space, Proc. Amer. Math. Soc. 59 (1976), 65-71. 
8. K. Knopp, Theorie und Anwendung der unendlichen Reihen, Springer-Verlag, Berlin and New York, 1964. MR 32 \# 1473.

9. P.-L. Lions, Approximation de points fixes de contractions, C. R. Acad. Sci. Paris Sér. A-B 284 (1977), A1357-A1359.

10. S. Reich, Approximating zeros of accretive operators, Proc. Amer. Math. Soc. 51 (1975), 381-384.

FAKUltÄt FÜR MATHEMATIK UND INFORMatik, UNIVERSITÄt MANNHEIM, D-6800 MANNheim, Federal Republic of Germany 